\title{
Epithelioid sarcoma of the tongue
}

\author{
X Leroy, A Delobelle, J L Lefebvre, V Cabaret, F Bloget, M O Vilain
}

\begin{abstract}
A case of epithelioid sarcoma in the tongue is reported. The patient, a 35 year old woman, presented with a nonulcerated painful lesion of the tongue. Microscopically, the tumour was characterised by multiple coalescent nodules with central geographic necrosis infiltrating the lingual muscle. The tumour cells were epithelioid with abundant eosinophilic cytoplasm and atypical nuclei. Immunohistochemically, the tumour cells stained for vimentin, keratin, and epithelial membrane antigen. These morphological and immunohistochemical appearances led to the diagnosis of epithelioid sarcoma of the tongue. Seven years later, the patient died with metastatic dissemination to the scalp, lungs, and brain. No case of epithelioid sarcoma arising in the tongue has been described previously. (F Clin Pathol 1997;50:869-870)
\end{abstract}

Keywords: epithelioid sarcoma; tongue; prognosis; immunohistochemistry

Epithelioid sarcoma is a rare malignant neoplasm occurring usually in the extremities of young adults. We present the first case in the literature of a primary epithelioid sarcoma located in the tongue, and demonstrate the use of immunohistochemistry in the differential diagnosis.

\section{Case report}

A 35 year old woman without a history of cancer presented with a painful non-ulcerated nodular lesion in the left half of the tongue of several months duration. A biopsy showed a sarcomatous proliferation. A hemiglossectomy was performed, followed by postoperative brachytherapy despite tumour free margins. Three years later, bilateral cervical lymph node metastases were removed. The patient died four years later with metastatic dissemination to the scalp, lungs, and brain in spite of radiation therapy.

\section{Pathological findings}

A $2.5 \mathrm{~cm}$ firm mass of the tongue was noted. Histologically, the tumour was poorly defined in the lingual muscle, typified by multiple nodules sometimes coalescent with central necrosis, which often appeared geographic (fig 1). The tumour cells were either fusiform or epithelioid, polygonal with a plump eosinophilic cytoplasm (fig 2). The nuclei were vesicular and sometimes had prominent nucleoli. Mitotic activity was high (25 mitotic fig- ures per 10 high power fields). No vascular invasion was seen. The lymph node metastases were similar to the primary tumour.

Immunohistochemically the tumour cells stained for cytokeratin (KL1 diluted 1/120; Immunotech, Marseille, France) (fig 3), epithelial membrane antigen diluted 1/50 (Dako, Trappe, France), and vimentin diluted 1/200 (Dako). No staining was seen with antibodies to S-100 protein diluted $1 / 400$ (Dako), cytokeratin 7 diluted 1/100 (Dako) or CD34 diluted $1 / 250$ (Immunotech). Therefore, this lesion was diagnosed as an epithelioid sarcoma of the tongue.

\section{Discussion}

Since the first description in 1970 by Enzinger, ${ }^{1}$ epithelioid sarcoma has been known to involve the superficial or deep soft tissues in

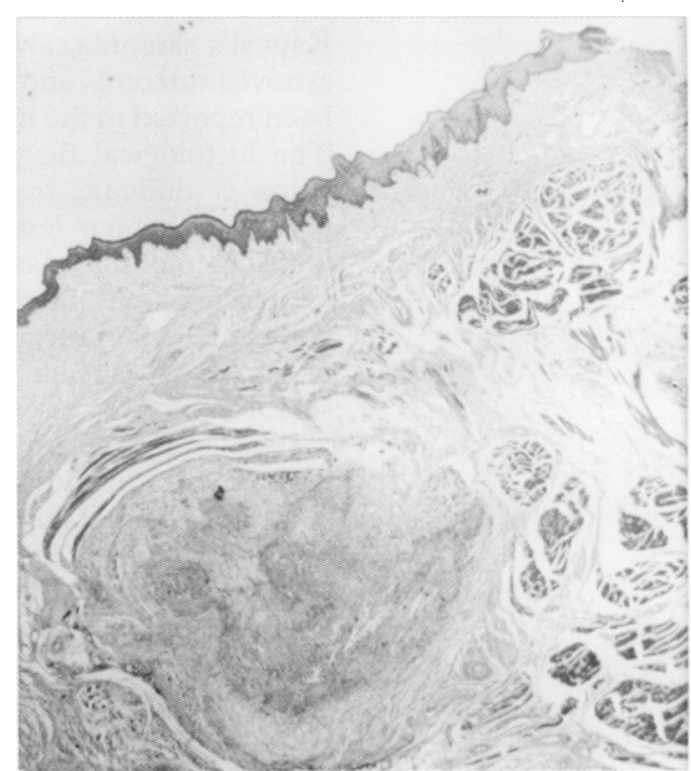

Figure 1 Nodular lesions infiltrating the lingual muscle.

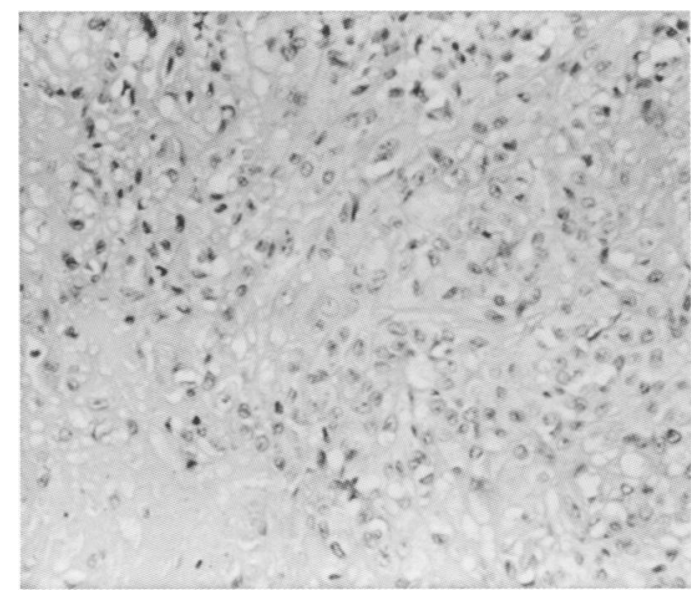

Figure 2 Typical epithelioid cells with central necrosis. 


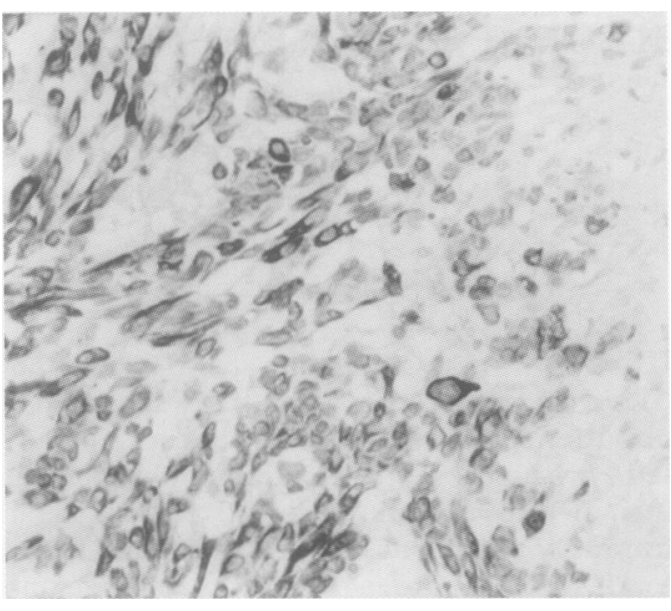

Figure 3 High positivity of cytokeratin in tumour cells (KL1).

the extremities (hands, fingers) of adolescents and young adults, more frequently in men than women. Rare cases in the literature are reported in the vulva or the penis. In the oral cavity, a case in the hard palate was presented by Jameson et $a .^{2}$

We describe the first case, to our knowledge, of primary epithelioid sarcoma of the tongue. Tongue sarcomas are very infrequent-fewer than $1 \%$ of all lingual neoplasms. Other than Kaposi's sarcoma, alveolar soft part sarcoma, synovial sarcoma, and osteogenic sarcoma have been reported in the literature as isolated cases. The histological diagnosis of epithelioid sarcoma is difficult, frequently being misinterpreted as benign lesions (necrobiotic granuloma or nodular fasciitis) or as malignant neoplasms (carcinoma, epithelioid angiosarcoma, malignant melanoma, malignant peripheral nerve sheath tumour or synovial sarcoma). ${ }^{13}$

Immunohistochemistry is helpful in the differential diagnosis. Epithelioid sarcoma shows a co-expression of keratin and vimentin; S-100 protein, CD 31, and HMB-45 are usually negative. Some epithelioid sarcomas are vimentin negative and may express CD $34{ }^{4}$ Expression of carcinoma embryonic antigen and actin is sometimes reported. In a recent study, Hazelbag et al ${ }^{5}$ showed the expression of different subtypes of cytokeratins: CK7 was negative as in our case. This profile was different in biphasic synovial sarcoma with a high positivity of CK7. Necrobiotic granuloma is ruled out by the positivity of CD68 and the negativity of keratin. Epithelioid angiosarcoma may express keratin but CD31 is also positive. Melanoma and epithelioid malignant periph- eral nerve sheath tumours are S-100 protein positive.

Ultrastructurally, the tumour cells of epithelioid sarcoma show intercellular junctions and abundant intermediate filaments. Several morphological variants of epithelioid sarcoma have recently been described: a fibroma-like variant, ${ }^{6}$ a chondroid variant, ${ }^{7}$ an angiomatoid form mimicking angiosarcoma, ${ }^{3}$ and a proximal-type with large cells and rhabdoid features that represents a more aggressive variant of epithelioid sarcoma. ${ }^{8}$

The histogenesis of this tumour is uncertain: a pluripotential mesoderm origin is proposed by some authors. ${ }^{9}$ A relation with extrarenal malignant rhabdoid tumours was also suggested. ${ }^{10}$

Epithelioid sarcoma is a malignant neoplasm with frequent local recurrences and often metastatic dissemination in regional lymph nodes, in the scalp, lungs, and brain.

Halling et $a l^{11}$ in a recent review of 55 cases found three histological variables associated with a poor prognosis: vascular invasion, tumour size of more than $5 \mathrm{~cm}$, and more than $30 \%$ necrosis.

The treatment is radical surgery with radiation therapy; however, $50 \%$ of patients develop metastatic lesions and the five year survival rate is $70 \% .^{11}$

We thank Dr J M Coindre and the French sarcoma group for reviewing the histological slides.

1 Enzinger FM. Epithelioid sarcoma. A sarcoma simulating a granuloma or a carcinoma. Cancer 1970;5:1029-41.

2 Jameson CF, Simpson MT, Towers JF. Primary epithelioid sarcoma of the hard palate. A case report. Int f Oral Maxilsarcoma of the hard palate.
lofac Surg 1990;19:240-2.

3 Von Hochstetter AR, Meyer VE, Grant JW, Honegger HP Schreiber A. Epithelioid sarcoma mimicking angiosarcoma: the value of immunohistochemistry in the differential diagnosis. Virchows Archiv A Pathol Anat 1991 418:271-8.

4 Arber DA, Kandalaft PL, Mehta P, Battifora H. Vimentinnegative epithelioid sarcoma. Am F Surg Pathol 1993;17: 302-7.

5 Hazelbag HM, Mooi WJ, Fleuren GJ, Hogendoorn PCW. Chain-specific keratin profile of epithelioid soft-tissue sarcomas. Appl Immunohistochem 1996;4:176-83.

6 Mirra JM, Kessler S, Butha S, Eckardt J. The fibroma-like variant of epithelioid sarcoma. A fibrohistiocytic/myoid cell lesion often confused with benign and malignant spindle lesion often confused with benign and

7 Chetty R, Slavin JL. Epithelioid sarcoma with extensive chondroid differentiation. Histopathology 1994;24:400-1.

8 Guillou L, Wadden C, Coindre JM, Krausz T, Fletche CDM. "Proximal-type" epithelioid sarcoma: a distinctive aggressive neoplasm showing rhabdoid features. $A m \mathcal{F}$ Surg Pathol 1997;21:134-46.

9 Manivel JC, Wick MR, Dehner LP, Sibley RK. Epithelioid sarcoma. An immunohistochemical study. Am f Clin Pathol 1987;87:319-26.

10 Molenaar WM, DeJong B, Dam-meiring A, Postma A DeVries J, Hoekstra HJ. Epithelioid sarcoma or malignant rhabdoid tumor of soft tissue? Hum Pathol 1989;20:34751 .

11 Halling AC, Wollan PC, Pritchard DJ, Vlasak R, Nascimento AG. Epithelioid sarcoma: A clinicopathologic review of 55 cases. Mayo Clin Proc 1996;71:636-42. 